Journal of Computer Science 7 (3): 352-358, 2011

ISSN 1549-3636

(C) 2011 Science Publications

\title{
An Effective Contention Aware Stable Path Finding Approach to Provide Quality of Service in Mobile Ad hoc Networks
}

\author{
${ }^{1}$ D.S. Thenmozhi and ${ }^{2} \mathrm{M}$. Rajaram \\ ${ }^{1}$ Department of Computer Science, Institute of Road and Transport Technology, \\ Erode, Tamilnadu, India \\ ${ }^{2}$ Anna University, Tirunelveli, Tamilnadu, India
}

\begin{abstract}
Problem statement: The application of Mobile Ad hoc NETworks (MANETs) has been increasing every day. Most applications that attract interest for use in current wired networks (e.g. video conferencing, on line movies and camera enabled instant messenger) would attract interest for MANETs also. Many applications that use MANETs include multimedia data that require Quality of Service (QoS) support for effective transmission. Basically meeting QoS is a mesh of multiple parameters. QoS routing based on multiple independent constraints has been proven to be NPcomplete. Hence, hardly any exact algorithms were proposed for this kind of problems. In this study we propose a new approach for QoS routing based on multiple constraints. Approach: QoS support can be achieved by finding a route satisfying the application requirements. This needs an admission control along the route to prevent new flows from consuming too many resources and disrupting the guarantees made to existing flows. In our work, the normal AODV is extended to perform QoS routing based on bandwidth requirement and link stability constraints. Link stability parameter is considered in an opportunistic way so that effective path meeting the required bandwidth and will last for complete session is established. Results: Simulation results show that our contention aware, link stability based route finding technique performs better than AODV in terms of throughput and control message overhead. It improves packet delivery ratio greatly without affecting the overall end-to-end throughput of existing flows. Conclusion: This multi constraint based AODV performs path finding with less overhead by adopting passive approach of listening to the medium. Hierarchical treatment of the metrics enables QoS robustness hence reduces subsequent route finding latency.
\end{abstract}

Key words: Quality of service, bandwidth estimation, contention aware, link stability, multiconstraints, Probability Distribution Function (PDF), Medium Access Control (MAC), simultaneous transmission, ad hoc networks, mobility model

\section{INTRODUCTION}

Ad hoc wireless network is made up of a group of mobile nodes and all communication is carried out through wireless medium in a distributed fashion without central controller. Nodes in MANETs are small radio devices with limited computational capacity and memory. The most desirable advantage of ad hoc networks is their easy and quick deployment. Routing in ad hoc networks has to adapt to the unexpected link breakage and topology changes. To discover and maintain the routes in ad hoc networks require more control traffic. This makes the task of performing ad hoc network routing more complex and less efficient.

A lot of work has been made on routing in ad hoc networks: the destination sequenced distance vector protocol, the wireless routing protocol, the temporally- ordered routing algorithms, the dynamic source routing protocol, the associativity based routing protocol and the zone routing protocol. Each routing protocol is suitable for the particular case of an ad hoc network and still has yet to be solved challenges (Jayakumar and Gopinath, 2007) like handling mobility, network scalability etc. These protocols tend to establish a path with least number of hops and only deal with the besteffort data traffic. Many of the applications, such as real-time video and voice are sensitive to the quality of service. Hence focus has been shifted from best-effort services to the provision of better defined QoS in ad hoc networks. In MANETs hard QoS (i.e., guaranteed throughput and delay) is difficult to achieve. Shared nature of the medium, unstable transmission links in MANETs need additional care at the time of quality of service support.

Corresponding author: D.S. Thenmozhi, Department of Computer Science, Institute of Road and Transport Technology, Erode, Tamilnadu, India 
In MANETs quality of service based routing is a relatively new problem. Cross layer multi constraint QoS routing is proposed in (Fan, 2004). In this study Fan proposes multi constraint routing based on MAC delay metric, link reliability and throughput constraints. In his work the QoS state for all paths must be discovered and kept fresh. The mechanism required for this is not discussed in the study. Power aware routing (Singh et al., 1998) is suggested by Rubin and Liu (2003) proposed QoS based on link stability. Link stability is mainly dependent on the node movement pattern. This study presents the Probability Distribution Function (PDF) of link lifetimes under various node movement models. The residual link lifetime is calculated as the area under the PDF for a given mobility model, taken between the links measured lifetime so far and infinity. Gupta et al. (2005) propose a heuristic interference aware QoS routing algorithm that chooses candidate paths based on localized information at the source node. Introducing a concept of clique graphs, which reflect links interfere with each other, thereby preventing simultaneous transmission. To enhance network scalability in MANETs proximity aware routing is presented by (Alandzi and Quintero, 2007) The protocol is framed to anticipate concave nodes and there by avoiding them. It helps to reduce the risks that the routing protocol will reach a concave node in the network. Lian et al. (2007) proposed a multi constraint QoS routing protocol with Route Request selection based on mobile prediction. In their strategy, a node forwards the RREQ packet to other nodes which are meeting link expiration time threshold. Link expiration time threshold value is calculated based on speed and direction of node movement.

A channel adaptive energy efficient MAC protocol, for efficient packet scheduling and queuing in ad hoc networks is proposed in (Priakanth and Thangaraj, 2009). The transmission is allowed only for those flows whose weight is greater than channel quality threshold. To avoid buffer overflow and achieve fairness for the poor quality nodes, a fair scheduling and queuing algorithm is designed where in the channel quality threshold is adjusted on the basis of current incoming traffic. For providing QoS guarantees and fairness among multiple users in wireless MAN, a two-layer scheduling algorithm has been proposed by Ghazizdeh et al. (Ghazizadeh et al., 2009). Importance of QoS in the forth coming $4 \mathrm{G}$ networks and the peculiar challenges to be solved are presented in (Quintero and Frutos, 2009). In (Thenmozhi and Lakshmipathi, 2010) the normal route finding method of AODV is improved as Quality of service Enhanced Ad hoc on Demand Distance Vector (QEAODV) routing. In this study QEAODV establishes a path between the source and the destination meeting the application stipulated throughput requirement. Contention which is the inherent problem of MANET is considered effectively in QEAODV. This extended AODV performs path finding with less overhead by adapting passive approach of listening to the medium. To support QoS for multimedia services in Wireless Metropolitan Area Network real time based scheduling approach is suggested in (Wu, 2010).

For most of the QoS required applications basic constraint is throughput (Hanzo and Tafazolli, 2007) i.e., number of bits per second. This is probably because assured throughput is somewhat of a "lowest common denominator" requirement. Not only for text files but also most voice or video applications require some level of guaranteed throughput in addition to other constraints. Hence one of the constraints that are considered in this study is 'throughput'. Other commonly employed QoS metrics (Hanzo and Tafazolli, 2007) are link stability, link reliability, endto-end delay, node buffer space, delay jitter, packet loss ratio. The parameter 'link stability' which is the major contributor for the prediction of expected route lifetime, is given due consideration in this study for QoS routing. To provide quality of service, the network considered must be combinatorially stable (Kuipers and VanMieghem, 2005).

A present trend that we have observed is that many researchers play great emphasis on the session admission capability of their protocol, which is admittedly most important but they often neglect the importance of session completion i.e. maintaining the routes and the QoS as long as the application data session requires. Ultimately the session completion is more important from a user perspective than session admission.

\section{MATERIALS AND METHODS}

Protocol description: AODV (Perkins et al., 2003) is a reactive routing technique used in ad hoc networks possessing the capabilities like low processing, memory overhead, low network utilization and it works well even in high mobility situation. The basic functionality of our multi constraint routing is much similar to the AODV protocol. Our extended AODV differs from normal AODV in the way the route discovery process is changed to provide quality of service support by performing multi constrained admission control at each node in the network. We focus on ad hoc networks based on single-channel MAC layers like IEEE 802.11. The main problem of the MANET comes from the shared nature of the wireless medium. The physical characteristics of wireless channels introduce the two challenges. First challenge is available bandwidth 
estimation at a node; second challenge is estimation of flow bandwidth requirement in a shared medium.

Available bandwidth estimation: The objective of admission control is to determine whether the available resources can meet the requirements of a new flow while maintaining bandwidth levels for existing flows. Each node views a different channel state. The available bandwidth in the network is not a local concept. To tackle this condition, two terms are introduced: local bandwidth available $\left(\mathrm{BW}_{\text {local }}\right)$, contention-neighbourhood bandwidth available $\left(\mathrm{BW}_{\mathrm{c}-}\right.$ neigh). Local bandwidth available is the amount of unconsumed bandwidth as observed by a given mode. Contention neighbourhood available bandwidth is the maximum amount of bandwidth that a node can use for transmission without affecting the reserved bandwidth of any existing flows in its carrier-sensing range.

In this study, admission control strategy involves two phases. In the first phase preliminary admission control is done. On receiving RREQ packet a node performs partial admission control. Application flow bandwidth requirement $\left(\mathrm{BW}_{\text {flow }}\right)$ is compared against node's local bandwidth available $\left(\mathrm{BW}_{\text {local }}\right)$ and contention neighbourhood bandwidth available $\left(\mathrm{BW}_{\mathrm{c}-}\right.$ neigh). $\mathrm{BW}_{\text {flow }}$ is calculated by considering only single node's transmission point of view. Bandwidth requirement of contention neighbours which are also involved in the same active flow are not considered at this stage. During the second phase of admission control known as final admission control node performs full fledged admission control. In this $\mathrm{BW}_{\text {local }}$ and $\mathrm{BW}_{\mathrm{c}-}$ neigh are compared against actual flow bandwidth requirement $\left(\mathrm{BW}_{\mathrm{a}_{-} \text {flow }}\right)$. $\mathrm{BW}_{\mathrm{a}_{\text {fllow }}}$ is computed based on the contention count $\left(\mathrm{C}_{\mathrm{ct}}\right)$ of the node.

Calculation of local bandwidth available $\left(\mathrm{BW}_{\text {local }}\right)$ : It is the unconsumed bandwidth at a given node. Each node in the MANET can determine its $\mathrm{BW}_{\text {local }}$ by passively listening network activities. In our approach, we use the fraction of channel idle time based on the past history as an indication of local available bandwidth at a node. By monitoring the amount of channel idle time, $T_{\text {idle }}$, during every period of time, $T_{p}$, the local bandwidth available $\mathrm{BW}_{\text {local }}$ of a node can be computed using a weighted average (Yang and Kravets, 2005) as follows:

$$
\mathrm{BW}_{\text {Iocal }}=\omega \mathrm{BW}_{\text {local }}+(1-\omega)\left(\frac{\mathrm{T}_{\text {idle }}}{\mathrm{T}_{\mathrm{p}}}\right) \mathrm{BW}_{\text {chamel }}
$$

where, $\mathrm{BW}_{\text {channel }}$ is the capacity of the channel and weight $\omega \varepsilon[0,1]$.
Calculation of contention neighbourhood bandwidth available $\left(\mathbf{B W}_{\text {c-neigh }}\right)$ Local bandwidth available information of a node cannot provide information about its contention neighbours since it does not know the amount of $\mathrm{BW}_{\text {local }}$ at other nodes. In our approach during the normal medium access using IEEE 802.11, node listens to the medium using a threshold value known as contention carrier sensing threshold. Normally carrier sensing range is twice the transmission range of a node. Contention carrier sensing threshold refers the range that covers the carrier sensing ranges of all of the sensing node's contention neighbours. Hence it is set to a value much lower than the carrier sensing threshold. When the signal strength of the carrier sensed by a node is smaller than the contention carrier sensing threshold there is no communication in its contention neighbourhood and contention neighbours of the node experience idle channels. The amount of time that the channel is in this idle state denoted as $\mathrm{T}_{\text {idle }}{ }^{\text {contention }}$, for every period of time $T_{p}$, contention neighbourhood available bandwidth $\mathrm{BW}_{\mathrm{c}_{-} \text {neigh }}$ is calculated using the following formula:

$$
\begin{aligned}
& \mathrm{BW}_{\mathrm{c}-\text { neigh }} \approx \omega \mathrm{BW}_{\mathrm{c} \text {-neigh }}+(1-\omega)\left(\frac{\mathrm{T}_{\text {idle }}^{\text {contention }}}{\mathrm{T}_{\mathrm{p}}}\right) \\
& \mathrm{BW}_{\text {channel }}
\end{aligned}
$$

where, $\mathrm{BW}_{\text {channel }}$ is the capacity of the channel and weight $\omega \varepsilon[0,1]$.

Calculation of Application's Flow Bandwidth

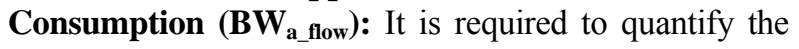
bandwidth that a new flow requires so that it can be decided whether the bandwidth available will satisfy the requirements of the flow. Foremost, the application's data rate has to be converted into the corresponding channel bandwidth requirement. As per IEEE 802.11, for every application data packet, the MAC layer performs handshaking. During this RTS, CTS and ACK control packets are involved. Hence each data packet's transmission time is calculated as follows:

$$
\mathrm{T}_{\text {data }}=\mathrm{T}_{\text {rts }}+\mathrm{T}_{\text {cts }}+\mathrm{T}_{\text {ack }}+\mathrm{T}_{\text {difs }}+3 \mathrm{~T}_{\text {sifs }}+\left(\frac{(\mathrm{P}+\mathrm{Q})}{\mathrm{BW}_{\text {channel }}}\right)
$$

Where:

$\mathrm{T}_{\text {data }}=$ Transmission time of each data packet

$\mathrm{T}_{\mathrm{rts}} \quad=$ Time for transmitting RTS

$\mathrm{T}_{\text {cts }} \quad=$ Time for transmitting CTS

$\mathrm{T}_{\text {ack }}=$ Time for transmitting ACK

$\mathrm{T}_{\text {difs }}=$ DCF inter frame space defined in the IEEE 802.11 protocol standard 
$\mathrm{T}_{\text {sifs }}=$ Short inter frame space defined in the IEEE 802.11 protocol standard

$\mathrm{P} \quad=$ Size of the data packet

$\mathrm{Q}=\mathrm{IP}$ and MAC packet header length

$\mathrm{BW}_{\text {channel }}=$ Channel capacity

If at every second, the application generates ' $R$ ' packets with average packet size ' $\mathrm{P}$ ', the corresponding channel bandwidth requirement is computed as follows:

$\mathrm{BW}_{\text {flow }}=\mathrm{R} \times \mathrm{T}_{\text {data }} \times \mathrm{BW}_{\text {channel }}$

Next factor to be considered is multiple nodes on the route of a new flow may contend for bandwidth at a single location. Every such node needs bandwidth equal to $\mathrm{BW}_{\text {flow. }}$ The number of such kind of nodes is known as contention count $\left(\mathrm{C}_{\mathrm{ct}}\right)$. Hence the bandwidth consumption of the flow at this location is expressed as:

$\mathrm{BW}_{\mathrm{a}_{-} \text {flow }}=\mathrm{C}_{\mathrm{ct}} \times \mathrm{BW}_{\text {flow }}$

Link stability prediction: In MANET some links are subject to have longer lifetime and some links are easily to be broken. Link stability parameter characterizes this feature. Link stability defines the possibility that the link happens to break. It is observed that in different network environments link stability follows certain statistical properties which enable this parameter to be measured or predicted. The movement pattern of the nodes in the ad hoc networks is a factor that has a considerable impact on network performance. Normally the applications resemble a Rayleigh distribution if the component factors are uncorrelated and distributed with equal variance. Ad hoc networks set in military and disaster relief situations, their node movements mostly follows random destination pattern. This study considers link stability property of node movements as per random destination model. This mobility model is found to produce a link lifetime PDF similar to a Rayleigh Distribution (Rubin and Liu, 2003). To measure the residual lifetime, the past link lifetime information must be considered. Its measurement model (Trivedi, 2008) is described as follows:

$\mathrm{P}($ residual_lifetime $>\mathrm{t})=\int \operatorname{Rayleigh}(\mathrm{r}) \mathrm{dr}$

To find the probability that a link's residual lifetime is greater than a time' $t$ ', the PDF of the link lifetime is integrated between ' $t+L_{p}$ ' and ' $\infty$ ', where ' $\mathrm{L}_{\mathrm{p}}$ ' is the link's past lifetime. Hence the cumulative distribution function is given as below:

$\mathrm{F}(\mathrm{r})=1-\mathrm{e}^{-\mathrm{r}^{2} / 2 \sigma^{2}}$ for $\mathrm{r} \varepsilon\left[\mathrm{t}=\mathrm{L}_{\mathrm{p}}, \infty\right]$
Route discovery process: During the route discovery process, the source broadcasts route request (RREQ) packet. Application's channel bandwidth requirement $\left(\mathrm{BW}_{\text {flow }}\right)(4)$ is computed by the source and included in the RREQ packet. The application will choose the required $\left(\mathrm{P}_{\mathrm{r} \text {-fail }}, \mathrm{T}_{\mathrm{r}}\right)$ pair. $\mathrm{P}_{\mathrm{r} \text {-fail }}$ represents the expected route break probability. It is a measure indicating that one of the links along the route may break during the whole flow time. $T_{r}$ represents the expected time duration of the flow. Link stability indicator $\left(\mathrm{P}_{\mathrm{r} \text {-fail }}, \mathrm{T}_{\mathrm{r}}\right)$ pair is also inserted in the RREQ packet. In addition to the above QoS indicators in the RREQ packet, another field $\mathrm{P}_{\mathrm{a}}$, which represents the accumulated survival probability of all the selected links from the source node to the current node is evaluated and added.

In multi constraint based AODV, each node computes $\mathrm{BW}_{\text {local }}$ and $\mathrm{BW}_{\mathrm{c}-\text { neigh }}$ as per (1) and (2) respectively. Also it calculates its residual lifetime probability. Every intermediate node, on receiving RREQ performs preliminary admission control as given in Fig. 1. We propose two different approaches to perform preliminary admission control. First approach known as S-approach both bandwidth and link stability requirements are given equal importance.

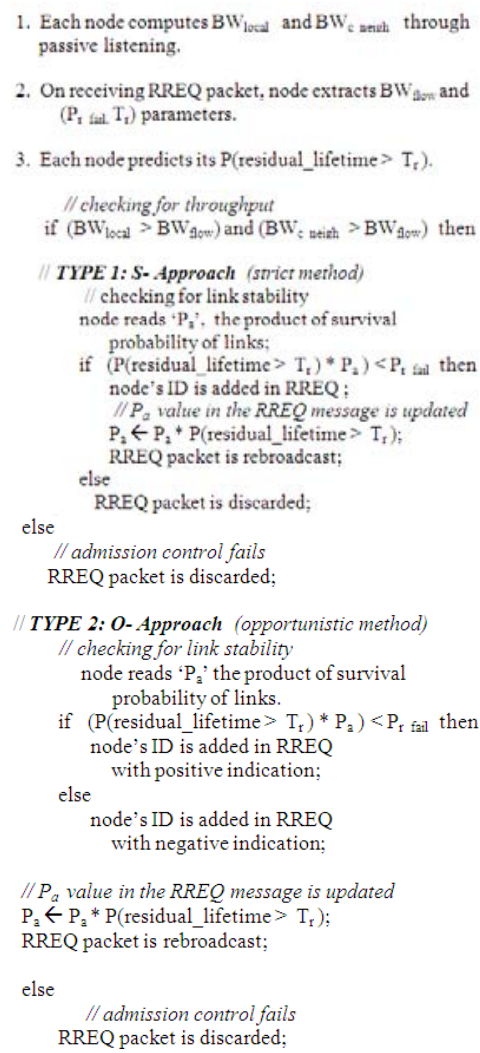

Fig. 1: Preliminary admission control algorithm 
On performing preliminary admission control node's ID is added in the RREQ if both the constraints are met (i.e., strict approach). Otherwise RREQ is simply discarded. Second approach known as O-approach (i.e. opportunistic approach), parameters are treated hierarchically. During preliminary admission control, node first checks for bandwidth requirement. If this constraint is met then it goes for checking link stability. If a node does not have requested bandwidth, preliminary admission control fails. While testing for link stability, the probabilistic prediction of the link stability value is used in an opportunistic way. If the stability requirement is met then node's ID is added with a positive indication, otherwise node's ID is added with negative indication.

In case of admission control failure, the RREQ is discarded. On success of the preliminary admission control the node sets up a reverse route entry in its routing table, adds its identifier in the RREQ packet. The value ' $\mathrm{P}_{\mathrm{a}}$ ', the survival probability is computed and updated in the RREQ packet. Then the node rebroadcasts the route request. Recording the sequence of hops in RREQ packet enables to determine the lower bound of the contention count of the complete route and also it can be used to eliminate circular routes.

When the intended destination receives the route request, it has the full route and sends a route reply (RREP) back to the source along the same route. The destination may get different routes. But all the routes partially satisfy the bandwidth requirement. As per Sapproach paths involving nodes meeting both the constraints are identified. Hence the priority is given to the path involving less number of hops. In O-approach the destination gives the preference to the route having all links possessing positive indication for the link stability. This way assures the sustained data transmission till the end of the flow. Next priority is given to the path involving less number of negative indication. The remaining routes are cached at the destination for certain time period in order to make use of them if final admission control fails for the first selected route. On receiving the RREP packet, a node performs final admission control as shown in Fig. 2. Node uses its contention count information at this stage.

On success of admission control, a soft reservation of bandwidth is made in the routing table and RREP is forwarded to its immediate predecessor. On failure of final admission control, admission failure message is sent to the destination via the same reverse route. Route error message (RERR) of AODV is modified to carry admission failure message on the reverse route. It enables cancellation of bandwidth reservation by the successor nodes. On receiving the admission failure message, the destination selects another fresh cached route and sends a RREP. On successfully receiving

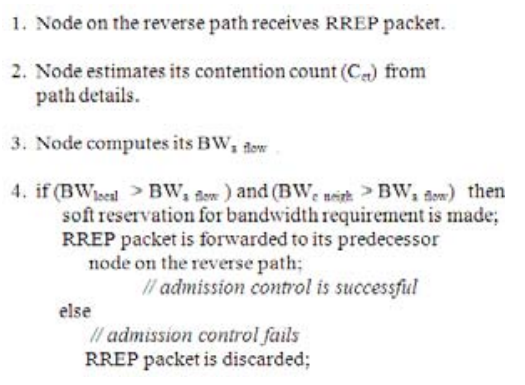

Fig. 2: Final admission control algorithm

RREP, a source has enough end-to-end bandwidth reserved on entire route. Also route is made up of sufficiently strong links. Communication can start at each node on the path; bandwidth reservation is refreshed by the arrival of data packets. The bandwidth reservation at the node automatically expires, if no data packet arrives due to link breakage.

\section{RESULTS}

Simulation: The multi constraint routing protocol is implemented using the NS-2 network simulator (McCanne et al., 1995) by modifying the basic AODV protocol. A modification is done in the MAC layer to capture the signal strength. The routing table is changed to hold the extra details like bandwidth reserved. Simulations are run for different scenarios created using 10, 20, 30, 40 and 50 nodes. Protocol evaluations are based on the simulation of wireless nodes forming an ad hoc network, moving about over a rectangle of size $1000 \times 1000 \mathrm{~m}$. Simulation time is $200 \mathrm{sec}$. At Medium Access Control (MAC) layer the 802.11 protocol is used. Radio transmission range of a node is set to $200 \mathrm{~m}$ and the carrier sensing range is set $400 \mathrm{~m}$. Each flow generated 10 packets per second. Each packet size is 512 bytes. The bandwidth of the channel is 2 Mbps. A number of simultaneous audio and video flows are made. Speed of nodes is varied from 2-10 m $\mathrm{sec}^{-1}$. Performance is assessed in terms of realizable throughput and control message overhead parameters. The performance of two approaches of multi constraint AODV (MC_AODV) is compared with normal AODV and also with QEAODV (Thenmozhi and Lakshmipathi, 2010) which is designed based on bandwidth constraint alone in terms of throughput and control message overhead.

\section{DISCUSSION}

Throughput of multi constraint AODV gets increased significantly when compared with normal 


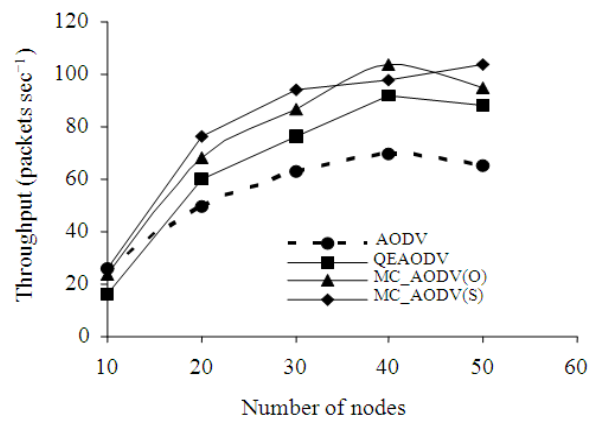

Fig. 3: Throughput of multi constraint AODV

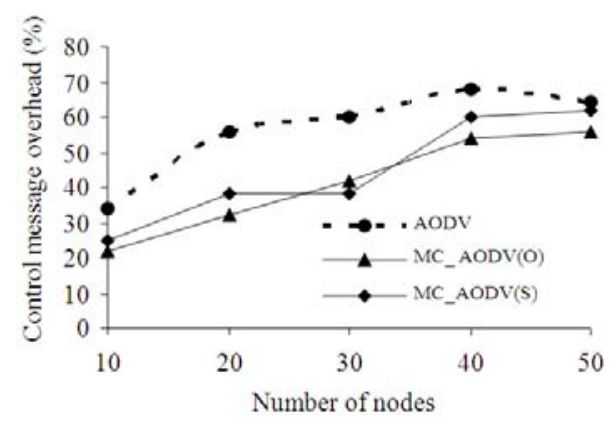

Fig. 4: Control message overhead for pause time $=10 \mathrm{sec}$

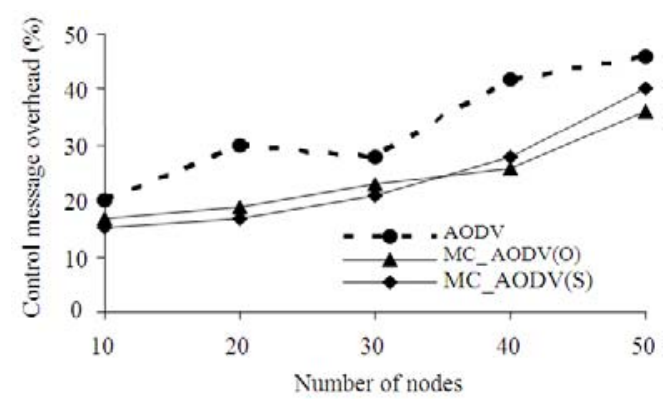

Fig. 5: Control message overhead for pause time $=20 \mathrm{sec}$

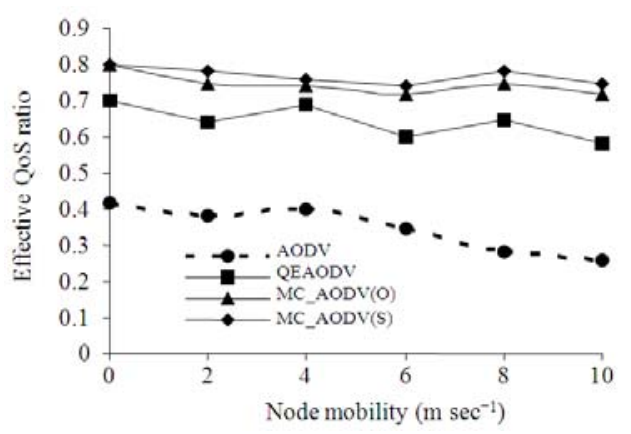

Fig. 6: QoS effectiveness for different node mobility values
AODV and single constraint based QoS routing (QEAODV) as shown in Fig. 3. From Fig. 4 and 5, it is inferred that control message overhead of multi constraint AODV execution is comparatively lower than normal AODV execution. Also as the nodes' pause time value increases, control message overhead decreases drastically. Also the path established based on S-approach is more effective than other methods. But this approach often reinitiates route discovery process. But O-approach avoids re-initiation of route discovery process by choosing the path with less number of negative marking. Figure 6 shows the QoS effectiveness with respect to node movement speed values. The QoS ratio is high when the mobility value is low. The QoS effectiveness decreases as the mobility ratio increases. The simulation result illustrates that by implementing link stability prediction our multi constraint AODV routing gives highly increased consistent QoS effectiveness.

\section{CONCLUSION}

In this study we presented multi constraint based routing technique to incorporate Quality of Service based applications in MANETs. Admission control during route discovery is mainly focused on throughput and link stability parameters. The concept of contention aware routing is performed by accounting contention neighbours bandwidth utilisation. Admission control process for a new flow involves two phases, so that reliable path can be achieved at the end. Our path finding approach is modified in such a way that it deals with common medium sharing problem of the ad hoc networks effectively. Inclusion of link stability metric ensures sustained QoS support till the complete data transmission. This multi constraint based AODV performs path finding with less overhead by adopting passive approach of listening to the medium. Hierarchical treatment of the metrics enables QoS robustness hence reduces subsequent route finding latency. Simulations show that contention aware, link stability based route finding technique performs better than AODV in terms of throughput and control message overhead. It improves packet delivery ratio greatly without affecting the overall end-to-end throughput of existing flows.

\section{REFERENCES}

Alandzi, V. and A. Quintero, 2007. Proximity aware routing in ad hoc networks. J. Comput. Sci., 3: 533-539. DOI: $10.3844 /$ jcssp.2007.533.539

Fan, Z., 2004. QoS routing using lower layer information in ad hoc networks. Proceedings of the International Symposium on Personal, Indoor and Mobile Radio Communications, Sept. 5-8, Bristol, UK., $\quad$ pp: $135-139 . \quad$ DOI: 10.1109/PIMRC.2004.1370851 
Ghazizadeh, R., P. Fan and Y. Pan, 2009. A two-layer channel-aware scheduling algorithm for IEEE 802.16 broadband wireless access systems. J. Applied Sci., 9: 449-458. DOI: 10.3923/jas.2009.449.458

Gupta, R., Z. Jia, T. Tung and J. Walrand, 2005. Interference-aware qos routing (IQRouting) for adhoc networks. Proceedings of the Global Telecommunications Conference, Dec. 2-2, St. Louis, MO., pp: 2599-2604. DOI: 10.1109/GLOCOM.2005.1578231

Hanzo, L. and R. Tafazolli, 2007. A survey of QoS routing solutions for mobile ad hoc networks. IEEE Commun. Surveys, 9: 50-57. DOI: 10.1109/COMST.2007.382407

Jayakumar, G. and G. Gopinath, 2007. Ad hoc mobile wireless networks routing protocols-a review. J. Comput. Sci., 3: 574-582. DOI: 10.3844/jcssp.2007.574.582

Kuipers, F. and P. VanMieghem, 2005. Conditions that impact the complexity of QoS routing. IEEE/ACM Trans. Network., 13: 717-730. DOI: 10.1109/TNET.2005.852882

Lian, J., L. Li and X. Zhu, 2007. A multi-constraint QoS routing protocol with route-request selection based on mobile predicting in MANET. Proceedings of the International Conference on Computational Intelligence and Security Workshops, Dec. 15-19, Harbin, pp: 342-345. DOI: 10.1109/CISW.2007.4425505

McCanne, S., S. Floyd, S. Fall and K. Varadhan, 1995. The Network Simulator -NS-2. The VINT Project. http://www.isi.edu/nsnam/ns

Perkins, C. and E. Belding-Royer and S. Das, 2003. Ad Hoc On-Demand Distance Vector (AODV) Routing. United States. http://portal.acm.org/citation.cfm?id=RFC3561
Priakanth, P. and P. Thangaraj, 2009. A channel adaptive energy efficient and fair scheduling media access control protocol for mobile adhoc networks. J. Comput. Sci., 5: 57-63. DOI: 10.3844/jcssp.2009.57.63

Quintero, A. and E.D. Frutos, 2009. MPLS based architecture for mobility and end-to-end QoS support in fourth generation mobile networks. J. Comput. Sci., 5: 255-262. DOI: 10.3844/jcssp.2009.255.262

Rubin, I. and Y.C. Liu, 2003. Link stability models for QoS ad hoc routing algorithms. Proceedings of the 58th IEEE Vehicular Technology Conference, Oct. 6-9, Los Angeles, CA, USA., pp: 3084-3088. DOI: 10.1109/VETECF.2003.1286191

Singh, S., M. Woo and C.S. Raghavendra, 1998. Power-aware routing in mobile ad hoc networks. Proceedings of the International Conference Mobile Computing and Networking, (ICMCN'98), ACM New York, NY, USA., pp: 181-190. DOI: 10.1145/288235.288286

Thenmozhi, D.S. and R. Lakshmipathi, 2010. Quality of service enhanced routing in mobile ad hoc networks. Int. J. Comput. Sci. Inform. Security, 8: 34-40.

Trivedi, K.S., 2008. Probability and Statistics with Reliability, Queueing and Computer Science Applications, 2nd Edn., Wiley India Pvt. Ltd., India, ISBN-10: 8126518537, pp: 848.

Wu, C.F., 2010. Real-time scheduling for multimedia services in IEEE 802.16 wireless metropolitan area network. Inform. Technol. J., 92: 1053-1067

Yang, Y. and R. Kravets, 2005. Contention-aware admission control for ad hoc networks. IEEE Trans. Mobil Comput., 4: 363-377. DOI: 10.1109/TMC.2005.52 